\title{
Cryopreservation of Persimmon Shoot Tips from Dormant Buds Using the D Cryo-plate Technique
}

\author{
Toshikazu Matsumoto ${ }^{1 *}$, Shin-ichi Yamamoto², Kuniaki Fukui ${ }^{2}$, Tariq Rafique ${ }^{2}$, \\ Florent Engelmann ${ }^{3}$ and Takao Niino ${ }^{4}$
}

\author{
${ }^{1}$ Faculty of Life and Environmental Science, Shimane University, Matsue 690-1102, Japan \\ ${ }^{2}$ National Institute of Agrobiological Sciences (NIAS), Tsukuba 305-8602, Japan \\ ${ }^{3}$ Institut de Recherche pour le Développement (IRD), UMR DIADE, 34394 Montpellier cedex 5, France \\ ${ }^{4}$ Gene Research Center, University of Tsukuba, Tsukuba 305-8572, Japan
}

Cryopreservation has become a very important tool for the long-term storage of plant germplasm. A new cryopreservation protocol based on air dehydration of explants placed on aluminum cryo-plates, termed the D cryo-plate technique, was developed. In this study, the most suitable conditions of cryopreservation for dormant shoot tips of Japanese persimmon (Diospyros kaki Thunb. 'Saijo') using the D cryo-plate technique were investigated. Dormant one-year-old shoots of persimmon were collected from the experimental farm of Shimane University in January 2013 and stored at $2^{\circ} \mathrm{C}$ until use. After surface sterilization, shoot tips of about $1 \mathrm{~mm}$ in size were dissected from the dormant buds and precultured overnight at $25^{\circ} \mathrm{C}$ on solidified $1 / 2 \mathrm{MS}$ medium containing $0.3 \mathrm{M}$ sucrose. Precultured shoot tips were placed on aluminum cryo-plates and embedded in calcium alginate gel. Osmoprotection of shoot tips was performed by immersing the cryo-plates for $30 \mathrm{~min}$ at $25^{\circ} \mathrm{C}$ in an $\mathrm{LS}$ solution containing $2 \mathrm{M}$ glycerol $+1.0 \mathrm{M}$ sucrose in $1 / 2 \mathrm{MS}$ solution. For the $\mathrm{D}$ cryo-plate technique, encapsulated shoot tips were dehydrated by placing the cryo-plates in the air current of a laminar flow cabinet for 30-90 min. Cooling was performed by placing the cryo-plates in uncapped cryotubes, which were immersed in liquid nitrogen. For rewarming, the cryo-plates were immersed in 1/2MS medium containing $1.0 \mathrm{M}$ sucrose for $20 \mathrm{~min}$ at $25^{\circ} \mathrm{C}$. In this study, the preculture did not improve the regrowth after cryopreservation; however, we consider that it should be performed in the D cryo-plate procedure for application of other cultivars and utilization in genebanks. A high regrowth rate of cryopreserved shoot tips $(84 \%)$ was achieved after dehydration for $30 \mathrm{~min}$. This optimized procedure was applied to 10 additional persimmon cultivars, resulting in regrowth rates ranging between 67 and $97 \%$, with an average of $87 \%$. As shoot tips derived from dormant buds proved to be highly tolerant to liquid nitrogen exposure, the D cryoplate technique may facilitate long-term conservation of persimmon germplasm.

Key Words: dormant shoot tips, genebank, kaki, osmoprotection.

\section{Introduction}

Diospyros includes about 400 species and is distributed throughout the tropics of Asia, Africa, and Central and South America. Only a few species are native to the temperate zone (Yonemori et al., 2000). These few spe-

Received; November 15, 2014. Accepted; January 6, 2015.

First Published Online in J-STAGE on March 12, 2015.

This study was mainly supported by a grant from the Ministry of Agriculture, Forestry and Fisheries of Japan (Genomics-based Technology for Agricultural Improvement, CRS-1001).

* Corresponding author (E-mail: tmatsumoto@life.shimane-u.ac.jp). cies are known as persimmon (Diospyros kaki Thunb.) and are cultivated mainly in Asian countries such as China, Japan, and Korea. Diospyros germplasm consists of many cultivars, which are conserved entirely in field genebanks (clonal orchards) in various repositories. However, field genebanks are not a desirable means of long-term conservation, especially for woody species, because of the high labor and financial costs involved and the problems related to disease and environmental stresses (Matsumoto et al., 2001).

Cryopreservation, a method of storage at super-low temperature such as in liquid nitrogen (LN), has been 
developed as a long-term conservation method for plant germplasm, which is utilized in genebanks of many countries (Sakai and Engelmann, 2007). Cryopreservation of persimmon shoot tips has been reported using slow cooling (Matsumoto et al., 2004), vitrification (Matsumoto et al., 2001; Niu et al., 2012) and dropletvitrification (Niu et al., 2012). However, the utilization of cryopreservation for persimmon germplasm is limited. Recently, cryopreservation protocols using aluminum cryo-plates have been reported as the $\mathrm{V}$ cryo-plate and the D cryo-plate techniques (Niino et al., 2013, 2014; Salma et al., 2014). The V cryo-plate method has been reported for strawberry, Dalmatian chrysanthemum, mint, mulberry, carnation, mat rush blueberry, and Perilla shoot tips/buds (Matsumoto et al., 2014a, b; Niino et al., 2013; Sekizawa et al., 2011; Yamamoto et al., 2011a, b, 2012a, b). The D cryo-plate method has been reported for mat rush shoot tips/buds (Niino et al., $2013,2014)$. Shoot tips adhere steadily to the cryoplates throughout the whole procedure for efficient performance. These protocols have two main advantages: they are user-friendly in terms of the procedure because samples held in aluminum plates are easy to handle during the procedure, and very high cooling and warming rates of samples held in these aluminum plates are possible by cooling by directly immersion in LN. As a result, very high regrowth has been obtained after cryopreservation with the materials tested (Niino et al., 2013). In the D cryo-plate method, large specimens consisting of buds covered with base sheaths and basal stems can be used for the materials, which is a practical and efficient method for cryopreservation (Niino et al., 2014). The keys to successful cryopreservation by the $\mathrm{D}$ cryo-plate technique are to induce the osmoprotection of shoot tips by preculture in sucrose and LS treatment and to determine the optimum dehydration time. In this study, we applied the D cryo-plate technique for cryopreserving shoot tips from dormant persimmon buds.

\section{Materials and Methods}

\section{Plant material}

One-year-old shoots $(70-100 \mathrm{~cm}$ in length) with dormant buds of Japanese persimmon (Diospyros kaki Thunb. 'Saijo') were collected from the experimental farm of Shimane University in mid-January 2013 and stored in plastic bags at $2^{\circ} \mathrm{C}$ for 1 to 3 months until use. One-year-old shoot sections ( $5 \mathrm{~cm}$ long) with two to three dormant buds each were washed with tap water and then sterilized in $70 \%$ ethanol for $2 \mathrm{~min}$ and in $2 \%$ sodium hypochlorite solution containing $0.01 \%$ Tween 20 for $30 \mathrm{~min}$. They were rinsed three times in sterile distilled water, and the shoot tips (about $1.0 \mathrm{~mm}$ in length, $1 \mathrm{~mm}$ base diameter) were dissected from the axillary vegetative buds. Ten additional persimmon cultivars were collected from Shimane Agricultural Experiment Station and tested for regrowth rates after cryopreservation by optimization of the D cryo-plate technique.

Cryopreservation of shoot tips using the D cryo-plate procedure

Excised shoot tips were cryopreserved using the D cryo-plate technique developed by Niino et al. (2013, 2014) with some modifications (Matsumoto et al., 2014a, b). The cryo-plates No. 2 (Yamamoto et al., 2011b) used were obtained from the National Institute of Agrobiological Sciences, Japan (Tsukuba, Japan).

The D cryo-plate protocol employed was as follows:

1) Excised shoot tips were precultured on solidified half-strength MS (Murashige and Skoog, 1962) medium (1/2MS) with $0.3 \mathrm{M}$ sucrose for 1 night at $25^{\circ} \mathrm{C}$.

2) Aluminum cryo-plate (Fig. 1) was placed in a Petri dish $(8 \mathrm{~cm}$ in diameter) and $2.0-2.5 \mu \mathrm{L}$ of $2 \%$ $(\mathrm{w} / \mathrm{w}) \mathrm{Na}$-alginate solution with $0.4 \mathrm{M}$ sucrose in $1 / 2 \mathrm{MS}$ medium was dispensed in each well.

3) Precultured shoot tips were placed in each well and a $100 \mathrm{mM} \mathrm{CaCl}{ }_{2}$ solution with $0.4 \mathrm{M}$ sucrose in $1 / 2 \mathrm{MS}$ was poured on the aluminum plates for $15 \mathrm{~min}$ for polymerization.

4) After removing the $\mathrm{CaCl}_{2}$ solution, the cryo-plate with shoot tips was treated with LS solution $(2 \mathrm{M}$ glycerol + 1 M sucrose; Yamamoto et al., 2011b) in a Petri dish for $30 \mathrm{~min}$ at $25^{\circ} \mathrm{C}$.

5) After the LS treatment, the cryo-plates with shoot tips were dehydrated under a laminar flow cabinet (SANYO Electric Co., Ltd., Tokyo, Japan). In this step, shoot tips were dehydrated for 30,60 , or $90 \mathrm{~min}$ at $25^{\circ} \mathrm{C}$. Then, the regrowth rates during the regeneration treatment in step 7 were compared. In the laminar flow cabinet, the air current velocity was approximately $0.6 \mathrm{~m} \cdot \mathrm{s}^{-1}$, while the temperature and humidity were $25^{\circ} \mathrm{C}$ and approximately $32 \%$, respectively.

6) Then, the cryo-plates were transferred into uncapped $2 \mathrm{~mL}$ cryotubes held on a cryo-cane, which were directly immersed in $\mathrm{LN}$ for at least $30 \mathrm{~min}$.

7) For rapid warming and unloading, cryo-plates with shoot tips in LN were transferred to $1 \mathrm{M}$ sucrose solution with $1 / 2 \mathrm{MS}$ in Petri dishes for $15 \mathrm{~min}$ at $25^{\circ} \mathrm{C}$. For plant regeneration, shoot tips were plated on solidified $1 / 2 \mathrm{MS}$ medium with $3 \%$ sucrose

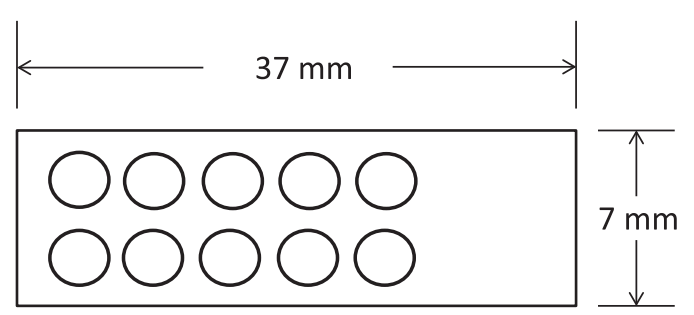

Fig. 1. Aluminum cryo-plate No. 2. Size: $7 \mathrm{~mm} \times 37 \mathrm{~mm} \times 0.5 \mathrm{~mm}$ with ten wells (diameter $1.5 \mathrm{~mm}$, depth $0.75 \mathrm{~mm}$ ). 
containing benzylaminopurine (BA) at $1 \mathrm{mg} \cdot \mathrm{L}^{-1}$ and incubated at $25^{\circ} \mathrm{C}$ under white fluorescent light $\left(50 \mu \mathrm{mol} \cdot \mathrm{s}^{-1} \cdot \mathrm{m}^{-2}\right)$ with a $16 \mathrm{~h}$ photoperiod for in vitro persimmon culture.

Optimization of the D cryo-plate technique and its application

Preculture and LS treatment

To optimize the protocol for shoot tips of persimmon, the regrowth of shoot tips was evaluated in the D cryoplate protocol with or without preculture on solidified $1 / 2 \mathrm{MS}$ with $0.3 \mathrm{M}$ sucrose for 1 night at $25^{\circ} \mathrm{C}$ and/or LS treatment of $2 \mathrm{M}$ glycerol $+1 \mathrm{M}$ sucrose solution for $30 \mathrm{~min}$ at $25^{\circ} \mathrm{C}$.

Application for other persimmon cultivars

To compare the regrowth after cryopreservation by the D cryo-plate technique under optimized conditions, 10 additional persimmon cultivars classified in terms of the nature of astringency loss of the fruit: PCA type 'Atago'; PVA types 'Koshuhyakume', 'Hiratanenashi', and 'Tonewase'; and PVNA types 'Fuyu', 'Kanshu', 'Maekawajiro', 'Shinshu', 'Taishu', and 'Taiten', were used.

Three replicates of about 10 shoot tips were tested in each experiment. Statistical analyses were performed using Tukey's test or two-way ANOVA.

\section{Results and Discussion}

Cryopreservation techniques are now used for plant germplasm storage at several institutes around the world (Niino, 2006). Cryopreservation has become an important tool for the long-term storage of plant germplasm and of experimental materials that possess unique attributes in order to minimize space and maintenance requirements without causing genetic alterations (Sakai, 1997). During cryopreservation, all biochemical activities are significantly decreased and all cellular divisions and metabolic processes stop (Engelmann, 2004; Kaviani, 2011). In our previous study, regrowth did not differ significantly between 10 years and 2 hours after cryopreservation, and regenerated wasabi (Wasabia japonica Matsumura) plants also showed no significant difference in regrowth and the results of morphological, biochemical and molecular analyses (Matsumoto et al., 2013). Therefore, cryopreservation is recognized to be the only technique currently available to ensure the safe and cost-efficient longterm conservation of these different types of germplasm (Engelmann, 2004). Recently, the D cryo-plate technique was developed as a new cryogenic method (Niino et al., 2013). This technique combines encapsulationdehydration and use of the V cryo-plate (Niino et al., 2013). Encapsulation-dehydration is a very efficient cryopreservation technique, which is simple and userfriendly to implement and allows problems associated with sensitivity of plant material to PVS2 vitrification solution to be overcome (Engelmann et al., 2008). In this method, the critical factors for obtaining high-level regrowth after cryopreservation are desiccation duration and osmo-protection treatment of shoot tips. The effect of desiccation duration on persimmon shoot tips is indicated in Table 1 . The highest regrowth $(84.3 \%)$ was obtained for $30 \mathrm{~min}$ desiccation. Additional desiccation tended to result in lower regrowth. To induce osmotolerance for shoot tips, the effects of two cryoprotection treatments, $0.3 \mathrm{M}$ sucrose preculture for 1 night and LS treatment with $2 \mathrm{M}$ glycerol $+1 \mathrm{M}$ sucrose solution, on shoot regrowth after cryopreservation were investigated. A high regrowth rate (over 90\%) was obtained following preculture + LS treatment and LS treatment without preculture (Table 2). Shoot tips that had not been submitted to the two treatments displayed $44.4 \%$ regrowth, while the effect of preculture on regrowth was not significant (49.5\% regrowth). Regrowth upon LS treatment was significantly increased $(93.0 \%)$, and that as the combined effect of preculture and LS treatment also significantly increased $(96.7 \%)$. These results mean that the LS treatment was necessary to increase the regrowth of persimmon shoot tips with the $\mathrm{D}$ cryo-plate technique; however, preculture was not always necessary for the D cryo-plate procedure. In addition, the interaction between preculture and LS treatment was not significant. On the other hand, the

Table 1. Effect of desiccation duration on regrowth of shoot tips of 'Saijo' persimmon cooled to $-196^{\circ} \mathrm{C}$ by $\mathrm{D}$ cryo-plate technique.

\begin{tabular}{cc}
\hline \hline Time of desiccation $(\mathrm{min})$ & Regrowth $(\% \pm \mathrm{SE})$ \\
\hline 30 & $84.3 \pm 7.9 \mathrm{a}$ \\
60 & $76.9 \pm 0.9 \mathrm{ab}$ \\
90 & $58.5 \pm 6.0 \mathrm{~b}$ \\
\hline
\end{tabular}

Preculture for $1 \mathrm{night}+\mathrm{LS}$ ( $2 \mathrm{M}$ glycerol $+1 \mathrm{M}$ sucrose) for $30 \mathrm{~min}$. Shoot tips were dissected from dormant buds.

Data followed by different letters are significantly different at $P<0.05$ (by Tukey's test).

Table 2. Effect of preculture and LS treatment on the regrowth of shoot tips of 'Saijo' persimmon cooled to $-196^{\circ} \mathrm{C}$ by D cryo-plate technique.

\begin{tabular}{|c|c|c|}
\hline Preculture ${ }^{\mathrm{z}}$ & LS treatment $\mathrm{t}^{\mathrm{y}}$ & Regrowth $(\% \pm \mathrm{SE})$ \\
\hline - & - & $44.4 \pm 5.6$ \\
\hline+ & - & $49.5 \pm 10.5$ \\
\hline - & + & $93.0 \pm 3.5$ \\
\hline+ & + & $96.7 \pm 3.3$ \\
\hline \multirow[t]{3}{*}{ Significance $^{\mathrm{x}}$} & Preculture & NS \\
\hline & LS treatment & $* *$ \\
\hline & Interaction & NS \\
\hline
\end{tabular}

Desiccation for $30 \mathrm{~min}$. Shoot tips were dissected from dormant buds.

z $0.3 \mathrm{M}$ sucrose for 1 night.

y $2 \mathrm{M}$ glycerol+1 $\mathrm{M}$ sucrose for $30 \mathrm{~min}$.

${ }^{x}$ NS and ** indicate not significant or significant at $P<0.01$ by $\operatorname{ANOVA}(\mathrm{n}=3)$. 
Table 3. Regrowth of cryopreserved shoot tips from 10 cultivars of persimmon by $\mathrm{D}$ cryo-plate technique.

\begin{tabular}{clc}
\hline \hline Astringent type $^{\mathrm{z}}$ & \multicolumn{1}{c}{ Cultivars } & Regrowth $(\% \pm \mathrm{SE})$ \\
\hline PCA & Atago & $95.8 \pm 4.2$ \\
PVA & Hiratanenashi & $88.9 \pm 6.4$ \\
& Koshuhyakume & $88.9 \pm 5.6$ \\
& Tonewase & $96.7 \pm 3.3$ \\
PCNA & Fuyu & $91.9 \pm 4.2$ \\
& Kanshu & $68.9 \pm 1.1$ \\
& Maekawajiro & $96.7 \pm 3.3$ \\
& Shinshu & $66.7 \pm 6.7$ \\
& Taishu & $82.6 \pm 9.0$ \\
& Taiten & $93.3 \pm 3.3$ \\
\hline & Average & 87.0 \\
\hline
\end{tabular}

Preculture for $1 \mathrm{night}+\mathrm{LS}$ treatment ( $2 \mathrm{M}$ glycerol $+1 \mathrm{M}$ sucrose) for $30 \mathrm{~min}$. Dessication for $30 \mathrm{~min}$. Shoot tips were dissected from dormant buds.

${ }^{z}$ PCA (pollination constant astringent), PVA (pollination variant astringent), PCNA (pollination constant non-astringent).

preculture on solidified medium with a high sucrose concentration for shoot tips was very effective to induce osmo-tolerance for the cryopreservation of many plants (Engelmann et al., 2008; Sakai et al., 2008). Moreover, in terms of the time schedule using the D cryo-plate procedure for utilization in genebanks, shoot tips/buds are dissected and precultured overnight at $25^{\circ} \mathrm{C}$ on medium with $0.3 \mathrm{M}$ sucrose on the first day, and shoot tips/ buds are plated on cryo-plates and polymerized, and then continued to the procedure (LS treatment, dehydration, etc.) on the second day (Niino et al., 2014). This preculture did not improve the regrowth after cryopreservation; however, we consider that it should be performed in the D cryo-plate procedure for application of other cultivars and utilization in genebanks.

Ten additional cultivars of temperate persimmon were tested using the optimal conditions established. Their regrowth rate ranged between 66.7 and $96.7 \%$, with an average of $87 \%$ (Table 3). The cryopreserved shoot tips resumed growth and developed normal shoots without callus formation (Fig. 2). To obtain high regrowth after cryopreservation, avoiding damage to explants during the cryogenic procedure or cooling/ warming is very important. In the vitrification technique using PVS2, shoot tips can be damaged by the chemical toxicity of PVS2, by osmotic stress due to excessive PVS2 treatment duration and by excision (Sakai et al., 2008). Avoiding the use of PVS2, using materials with higher moisture content and performing minimal excision of young leaves and/or sheaths may limit damage to specimens (Niino et al., 2013). The D cryo-plate technique allows the problems associated with sensitivity to PVS2, insufficient or excessive dehydration, and damage to and loss of material during excision and manipulation to be overcome (Niino et al., 2013). Niino et al. (2013) also reported the advantages of the D cryo-

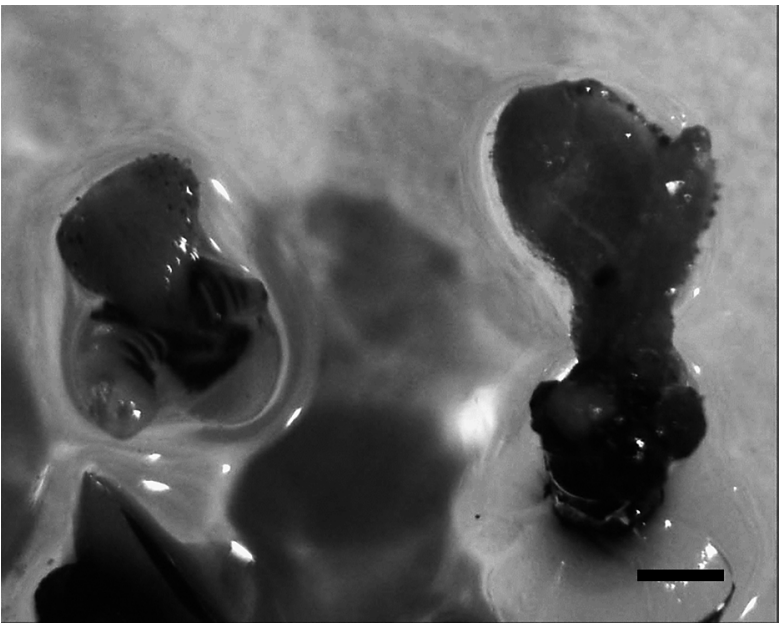

Fig. 2. Regrowth of cryopreserved shoot tips of 'Saijo' persimmon by $\mathrm{D}$ cryo-plate technique 25 days after rewarming. Scale bar: $1 \mathrm{~mm}$.

plate technique, including the use of larger specimens, the production of very high regrowth and much less laborious procedures than for other methods. In this study, the regrowth rate of shoot tips sampled from dormant buds of a total of 11 cultivars cryopreserved using the D cryo-plate technique was very high. Dormant buds of persimmon display very high cold tolerance (Matsumoto et al., 2001). Moreover, they are covered with scales and several young leaves, and can thus tolerate strong sterilization treatment (Matsumoto et al., 2004) with very low contamination.

In this study, we demonstrated that the $\mathrm{D}$ cryo-plate technique led to very high regrowth with a total of 11 persimmon cultivars and that shoot tips sampled from dormant buds represent very good material for cryopreservation. Therefore, these established protocols appear to be promising for the cryopreservation of other cultivars and species of Diospyros plants, with some modifications.

\section{Acknowledgements}

The authors wish to express their thanks to Shimane Agricultural Experiment Station for the gifts of ten cultivars of one-year-old persimmon shoots with dormant buds.

\section{Literature Cited}

Engelmann, F. 2004. Plant Cryopreservation: Progress and prospects. In Vitro Cell. Dev. Biol. 40: 427-433.

Engelmann, F., M. T. Gonzalez-Arnao, Y. Wu and R. Escobar. 2008. The development of encapsulation dehydration. p. 5975. In: B. M. Reed (ed.). Plant Cryopreservation: A Practical Guide. Springer, New York.

Kaviani, B. 2011. Conservation of plant genetic resources by cryopreservation. Aust. J. Crop Sci. 5: 778-800.

Matsumoto, T., T. Akihiro, S. Maki, K. Mochida, M. Kitagawa, D. Tanaka, S. Yamamoto and T. Niino. 2013. Genetic stability assessment of wasabi plant regenerated from long-term 
cryopreserved shoot tips using morphological, biochemical and molecular analysis. CryoLett. 34: 128-136.

Matsumoto, T., K. Mochida, H. Itamura and A. Sakai. 2001. Cryopreservation of persimmon (Diospyros kaki Thunb.) by vitrification of dormant shoot tips. Plant Cell Rep. 20: 398402.

Matsumoto, T., T. Niino, K. Shirata, T. Kurahashi, S. Matsumoto, S. Maki and H. Itamura. 2004. Long-term conservation of Diospyros germplasm using dormant buds by a prefreezing method. Plant Biotechnol. 21: 229-232.

Matsumoto, T., T. Niino, S. Yamamoto and K. Fukui. 2014a. Cryopreservation of blueberry shoot tips using V-cryo-plate method. Abst. Amer. Soc. Hort. Sci. 2014 Annual Conference: $337-338$.

Matsumoto, T., K. Yoshimatsu, N. Kawahara, S. Yamamoto and T. Niino. 2014b. Development of in vitro propagation by node culture and cryopreservation by V-Cryo-plate method for Perilla frutescens. Adv. Hort. Sci. 28: 79-83.

Murashige, T. and F. Skoog. 1962. A revised medium for rapid growth and bioassays with tobacco tissue cultures. Physiol. Plant. 15: 473-487.

Niino, T. 2006. Developments in plant genetic resources cryopreservation technologies. p. 197-217. In: J. H. Kang (ed.). Effective Genebank Management in APEC Member Economies, NIAB, Suwon.

Niino, T., K. Watanabe, N. Nohara, T. Rafique, S. Yamamoto, K. Fukui, M. V. Arizaga, C. R. C. Martinez, T. Matsumoto and F. Engelmann. 2014. Practical cryopreservation of mat rush basal stem buds by air dehydration using aluminum cryoplate. Plant Biotechnol. 31: 281-287.

Niino, T., S. Yamamoto, K. Fukui, C. R. C. Martínez, M. V. Arizaga, T. Matsumoto and F. Engelmann. 2013. Dehydration improves cryopreservation of mat rush (Juncus decipiens Nakai) basal stem buds on cryo-plates. CryoLett. 34: 549-560.

Niu, Y., Z. Luo, Y. Zhang and Q. Zhang. 2012. Cryopreservation of in vitro grown shoot tips of Diospyros kaki Thunb. using different methods. CryoLett. 33: 69-74.

Sakai, A. 1997. Potentially valuable cryogenic procedures for cryopreservation of cultured plant meristems. p. 53-66. In: M. K. Lazdan and E. C. Cocking (eds.). Conservation of
Plant Genetic Resources In Vitro, Science Publishers, New York.

Sakai, A. and F. Engelmann. 2007. Vitrification, encapsulationvitrification and droplet-vitrification: A review. CryoLett. 28: $151-172$.

Sakai, A., D. Hirai and T. Niino. 2008. Development of PVSbased vitrification and encapsulation-vitrification protocols. p. 33-58. In: B. M. Reed (ed.). Plant Cryopreservation: A Practical Guide, Springer, New York.

Salma, M., L. Fkic, I. Engelmann-Sylvestrea, T. Niino and F. Engelmann. 2014. Comparison of droplet-vitrification and D-cryoplate for cryopreservation of date palm (Phoenix dactylifera L.) polyembryonic masses. Sci. Hortic. 179: 9197.

Sekizawa, K., S. Yamamoto, T. Rafique, K. Fukui and T. Niino. 2011. Cryopreservation of in vitro-grown shoot tips of carnation (Dianthus caryophyllus L.) by vitrification method using aluminum cryo-plates. Plant Biotechnol. 28: 401-405.

Yamamoto, S., F. Fukui, T. Rafique, N. I. Khan, C. R. Castillo Martinez, K. Sekizawa, T. Matsumoto and T. Niino. 2011a. Cryopreservation of in vitro-grown shoot tips of strawberry by the vitrification method using aluminum cryo-plates. Plant Genet. Resour. Charact. Util. 10: 14-19.

Yamamoto, S., T. Rafique, K. Fukui, K. Sekizawa, A. Koyama, T. Ichihashi and T. Niino. 2012a. Development of an effective cryopreservation protocol using aluminum cryo-plates for in vitro-grown shoot tips of mulberries (Morus spp.) originated from the tropics and subtropics. Sanshi-Konchu Biotec. 81: 57-62 (In Japanese with English abstract).

Yamamoto, S., T. Rafique, K. Fukui, K. Sekizawa and T. Niino. 2012b. V-Cryo-plate procedure as an effective protocol for cryobanks. Case study of mint cryopreservation. CryoLett. 33: $12-23$.

Yamamoto, S., T. Rafique, W. S. Priyantha, K. Fukui, T. Matsumoto and T. Niino. 2011b. Development of a cryopreservation procedure using aluminum cryo-plates. CryoLett. 32: 256-265.

Yonemori, K., A. Sugiura and M. Yamada. 2000. Persimmon genetics and breeding. p. 191-225. In: J. Janick (ed.). Plant Breeding Reviews 19, John Wiley \& Sons, New York. 